\title{
Constraints on the standard model extension with binary pulsars
}

\author{
Yi Xie \\ Department of Astronomy, Nanjing University, Nanjing 210093, China \\ email: yixie@nju.edu.cn
}

\begin{abstract}
Under the standard model extension (SME) framework, Lorentz invariance is tested in five binary pulsars: PSR J0737-3039, PSR B1534+12, PSR J1756-2251, PSR B1913+16 and PSR B2127+11C. By analyzing the advance of periastron, we obtain the constraints on a dimensionless combination of SME parameters that is sensitive to timing observations. The results imply no evidence for the break of Lorentz invariance at $10^{-10}$ level, one order of magnitude larger than previous estimation.
\end{abstract}

Keywords. gravitation, relativity, pulsars: PSR 0737-3039, PSR B1534+12, PSR J1756-2251, PSR B1913+16, PSR B2127+11C

\section{Introduction}

Unification of general relativity (GR) and quantum mechanics is a grand challenge in the fundamental physics. Some candidates of a self-consistent quantum theory of gravity emerge from tiny violations of Lorentz symmetry (Kostelecký 2005; Mattingly 2005). To describe observable effects of the violations, effective field theories could be a theoretical framework for tests.

The standard model extension (SME) is one of those effective theories. It includes the Lagrange densities for GR and the standard model for particle physics and allows possible breaking of Lorentz symmetry (Bailey \& Kostelecký 2006). The SME parameters $\bar{s}^{\mu \nu}$ control the leading signals of Lorentz violation in the gravitational experiments in the case of the pure-gravity sector of the minimal SME. By analyzing archival lunar laser ranging data, Battat et al. (2007) constrain these dimensionless parameters at the range from $10^{-11}$ to $10^{-6}$, which means no evidence for Lorentz violation at the same level.

However, tighter constraints on $\bar{s}^{\mu \nu}$ would be hard to obtain in the solar system because the gravitational field is weak there. Thus, for this purpose, binary pulsars provide a good opportunity. Because of their stronger gravitational fields, for example the relativistic periastron advance in the double pulsars could exceed the corresponding value for Mercury by a factor of $\sim 10^{5}$, these systems are taken as an ideal and clean test-bed for testing GR, alternative relativistic theories of gravity and modified gravity, such as the works by Bell et al. (1996), Damour \& Esposito-Farèse (1996), Kramer et al. (2006), Deng (2009) and Deng (2011).

Motivated by this advantage of binary pulsars, we will try to test Lorentz invariance under the SME framework with five binary pulsars: PSR J0737-3039, PSR B1534+12, PSR J1756-2251, PSR B1913+16 and PSR B2127+11C. In Section 2, the orbital dynamics of double pulsars in the SME will be briefed. Observational data will be used to constrain the SME parameters in Section 3. The conclusions will be presented in Section 4. 


\section{Orbital dynamics of double pulsars in SME}

When the pure-gravity sector of the minimal SME is considered, it will cause secular evolutions of the orbits of double pulsars. Since timing observations of double pulsars could obtain its value very precisely, the periastron advance plays a much more important role in constraining $\bar{s}^{\mu \nu}$ and, with widely used notations in celestial mechanics, it reads (Bailey \& Kostelecký 2006)

$$
\begin{aligned}
\left.\left\langle\frac{d \omega}{d t}\right\rangle\right|_{\mathrm{SME}}= & -\frac{n}{\tan i\left(1-e^{2}\right)^{1 / 2}}\left[\frac{\varepsilon}{e^{2}} \bar{s}_{k P} \sin \omega+\frac{\left(e^{2}-\varepsilon\right)}{e^{2}} \bar{s}_{k Q} \cos \omega-\frac{\delta m}{M} \frac{2 n a \varepsilon}{e} \bar{s}_{k} \cos \omega\right] \\
& -n\left[\frac{\left(e^{2}-2 \varepsilon\right)}{2 e^{4}}\left(\bar{s}_{P P}-\bar{s}_{Q Q}\right)+\frac{\delta m}{M} \frac{2 n a\left(e^{2}-\varepsilon\right)}{e^{3}\left(1-e^{2}\right)^{1 / 2}} \bar{s}_{Q}\right]
\end{aligned}
$$

where $M=m_{1}+m_{2}, \delta m=m_{2}-m_{1}\left(m_{2}>m_{1}\right)$ and $\varepsilon=1-\left(1-e^{2}\right)^{1 / 2}$. In this expression, the coefficients $\bar{s}$. and $\bar{s}$.. for Lorentz violation with subscripts $P, Q$ and $k$ are projections of $\bar{s}^{\mu \nu}$ along the unit vectors $\boldsymbol{P}, \boldsymbol{Q}$ and $\boldsymbol{k}$. The unit vector $\boldsymbol{k}$ is perpendicular to the orbital plane of the binary pulsars, $\boldsymbol{P}$ points from the focus to the periastron, and $\boldsymbol{Q}=\boldsymbol{k} \times \boldsymbol{P}$. By definitions (Bailey \& Kostelecký 2006), $\bar{s}_{k} \equiv \bar{s}^{0 j} k^{j}, \bar{s}_{Q} \equiv \bar{s}^{0 j} Q^{j}, \bar{s}_{k P} \equiv \bar{s}^{i j} k^{i} P^{j}$, $\bar{s}_{k Q} \equiv \bar{s}^{i j} k^{i} Q^{j}, \bar{s}_{P P} \equiv \bar{s}^{i j} P^{i} P^{j}$ and $\bar{s}_{Q Q} \equiv \bar{s}^{i j} Q^{i} Q^{j}$. However, according to Eq. (2.1), it is easy to see that the measurement of $\dot{\omega}$ is sensitive to a combination of $\bar{s}^{\mu \nu}$ instead of its individual components. Bailey \& Kostelecký (2006) define the combination as

$$
\begin{aligned}
\bar{s}_{\omega} \equiv & \bar{s}_{k P} \sin \omega+\left(1-e^{2}\right)^{1 / 2} \bar{s}_{k Q} \cos \omega-\frac{\delta m}{M} 2 n a e \bar{s}_{k} \cos \omega \\
& +\tan i \frac{\left(1-e^{2}\right)^{1 / 2}\left(e^{2}-2 \varepsilon\right)}{2 e^{2} \varepsilon}\left(\bar{s}_{P P}-\bar{s}_{Q Q}\right)+\frac{m}{M} 2 n a \tan i \frac{\left(e^{2}-\varepsilon\right)}{e \varepsilon} \bar{s}_{Q},
\end{aligned}
$$

and crudely estimate its value at the level of $10^{-11}$.

Together with the contribution from GR, the total secular periastron advance of a double pulsars system is

$$
\begin{aligned}
\dot{\omega} & =3\left(\frac{P_{b}}{2 \pi}\right)^{-5 / 3}\left(\frac{G M}{c^{3}}\right)^{2 / 3}\left(1-e^{2}\right)^{-1}-\frac{n \varepsilon}{\tan i\left(1-e^{2}\right)^{1 / 2} e^{2}} \bar{s}_{\omega} \\
& =3\left(\frac{P_{b}}{2 \pi}\right)^{-5 / 3} T_{\odot}^{2 / 3}\left(\frac{M}{M_{\odot}}\right)^{2 / 3}\left(1-e^{2}\right)^{-1}-\frac{2 \pi \varepsilon s}{P_{b}\left(1-e^{2}\right)^{1 / 2} e^{2}\left(1-s^{2}\right)^{1 / 2}} \bar{s}_{\omega}
\end{aligned}
$$

where $T_{\odot} \equiv G M_{\odot} / c^{3}=4.925490947 \mu$ s and

$$
s=x\left(\frac{P_{b}}{2 \pi}\right)^{-2 / 3} T_{\odot}^{-1 / 3} M^{2 / 3} m_{2}^{-1} .
$$

The quantity $x$ in Eq. (2.4) is the projected semi-major axis, which is usually given by the timing observations, while, in some cases, $s$ could be measured directly so that there is no necessity to evaluate it from this equation. In this work, Eq. (2.3) will be taken to find the constraints on $\bar{s}_{\omega}$ with timing measurements of double pulsars.

\section{Observational constraints}

Long-term timing observations can determine the geometrical and physical parameters of binary pulsars very well. Among them, PSR J0737-3039 (Kramer et al. 2006), PSR B1534+12 (Stairs et al. 2002), PSR J1756-2251 (Faulkner et al. 2005), PSR B1913+16 (Weisberg et al. 2010) and PSR B2127+11C (Jacoby et al. 2006) are good samples for gravitational tests. Some of their timing parameters are listed in the Table 1. In terms 
Table 1. Timing Parameters of the Double Pulsars.

\begin{tabular}{lllllll}
\hline PSR & $P_{b}(\mathrm{~d})$ & $M\left(M_{\odot}\right) e$ & $s$ & $\dot{\omega}\left({ }^{\circ} \mathrm{yr}^{-1}\right)$ & Reference \\
\hline J0737-3039 & 0.10225156248 & 2.58708 & 0.0877775 & 0.99974 & $16.89947(68)$ & Kramer et al. $(2006)$ \\
B1534+12 & 0.420737299122 & 2.678428 & 0.2736775 & 0.975 & $1.755789(9)$ & Stairs et al. $(2002)$ \\
J1756-2251 & 0.319633898 & 2.574 & 0.180567 & $0.961^{a}$ & $2.585(2)$ & Faulkner et al. $(2005)$ \\
B1913+16 & 0.322997448911 & 2.828378 & 0.6171334 & $0.733650^{a}$ & $4.226598(5)$ & Weisberg et al. $(2010)$ \\
B2127+11C & 0.33528204828 & 2.71279 & 0.681395 & $0.76762^{a}$ & $4.4644(1)$ & Jacoby et al. $(2006)$ \\
\hline
\end{tabular}

${ }^{a}$ Derived value according to Eq. (2.4).

Table 2. Values of $\bar{s}_{\omega}$.

\begin{tabular}{ccc}
\hline Group I & Group II & $\begin{array}{c}\text { Predicted sensitivity } \\
\text { Bailey \& Kostelecký }(2006)\end{array}$ \\
\hline $\bar{s}_{\omega} \quad(-1.24 \pm 0.54) \times 10^{-10}$ & $(-1.42 \pm 0.75) \times 10^{-10}$ & $10^{-11}$ \\
\hline
\end{tabular}

of the estimated uncertainties given in parentheses after $\dot{\omega}$, the data pool is divided into two groups: Group I, all the double pulsars are taken; and Group II, including PSR B1913+16, PSR B1534+12 and PSR B2127+11C, which have the smallest uncertainties.

By weighted least square method, the parameter $\bar{s}_{\omega}$ is estimated (see Table 2). The estimation made by Group I is $\bar{s}_{\omega}=(-1.24 \pm 0.54) \times 10^{-10}$ and Group II gives $\bar{s}_{\omega}=$ $(-1.42 \pm 0.75) \times 10^{-10}$. For comparison, Bailey \& Kostelecký (2006) propose the attainable experimental sensitivity of $\bar{s}_{\omega}$ is $10^{-11}$, which is 10 times less than the results we obtain.

\section{Conclusions}

In this work, we test Lorentz violation with five binary pulsars under the framework of standard model extension. It finds that $\bar{s}_{\omega}$, which is a dimensionless combination of SME parameters, is at the order of $10^{-10}$, whether all five systems are taken or top three systems with the smallest estimated uncertainties of periastron advances are used. This value, one order of magnitude greater than the estimation by Bailey \& Kostelecký (2006), implies no evidence for the break of Lorentz invariance at $10^{-10}$ level.

\section{References}

Bailey, Q. G. \& Kostelecký, V. A. 2006, Phys. Rev. D, 74, 045001

Battat, J. B. R., Chandler, J. F., \& Stubbs, C. W. 2007, Phys. Rev. Lett., 99, 241103

Bell, J. F., Camilo, F., \& Damour, T. 1996, ApJ, 464, 857

Damour, T. \& Esposito-Farèse, G. 1996, Phys. Rev. D, 53, 5541

Deng, X. 2011, Science in China G: Physics and Astronomy, 54, 2071

Deng, X.-M., Xie, Y., \& Huang, T.-Y. 2009, Phys. Rev. D, 79, 044014

Faulkner, A. J., Kramer, M., Lyne, A. G., et al. 2005, ApJ, 618, L119

Jacoby, B. A., Cameron, P. B., Jenet, F. A., et al. 2006, ApJ, 644, L113

Kostelecký, V., ed. 2005, Third Meeting on CPT and Lorentz Symmetry (Singapore: World Scientific)

Kramer, M., Stairs, I. H., Manchester, R. N., et al. 2006, Science, 314, 97

Mattingly, D. 2005, Living Reviews in Relativity, 8, 5

Stairs, I. H., Thorsett, S. E., Taylor, J. H., \& Wolszczan, A. 2002, ApJ, 581, 501

Weisberg, J. M., Nice, D. J., \& Taylor, J. H. 2010, ApJ, 722, 1030 